\title{
ANÁLISE BIOMECÂNICA DOS MÚSCULOS EXTENSORES E FLEXORES DO JOELHO, POR MEIO DO DINAMÔMETRO ISOCINÉTICO, EM PRATICANTES DE ARTES MARCIAIS
}

\section{BIOMECHANIC ANALYSIS OF EXTENSION AND FLEXORES MUSCLES OF THE KNEE WITH AN ISOKINETIC DYNAMOMETER IN MARTIAL ARTS ATHLETES}

\author{
Susane Moreira Machado ${ }^{1}$ \\ Fernanda Maria Gonzaga Napoleone ${ }^{1}$ \\ Leandra Monteiro de Paiva ${ }^{2}$ \\ Newton Soares da Silva ${ }^{3}$ \\ Alderico de Paula de Lima $^{4}$ \\ Rodrigo Alexis Lazo Osorio ${ }^{5}$
}

RESUMO: As artes marciais Taekwondo e Kick Boxing utilizam o chute durante a competição, sobrecarregando a articulação do joelho que é solicitada para dar mobilidade e estabilidade e está em constante movimento e stress. A análise biomecânica em praticantes de artes marciais busca a compreensão e quantificação da sobrecarga mecânica imposta ao aparelho locomotor; para isso, há a avaliação isocinética. Este estudo consiste na análise biomecânica dos músculos extensores e flexores do joelho, por meio do dinamômetro isocinético, para a verificação da relação agonista/antagonista do joelho, na produção de torque, utilizando-se a velocidade de $60 \%$ s, e comparação do pico de torque, na potência muscular dos atletas, nas velocidades de $180 \%$ s e $240 \%$. Observou-se que, entre a musculatura agonista e antagonista do joelho, na velocidade de 60\%, uma média de $51,02 \%$ para o joelho esquerdo e 53,43\% para o direito, apresentou um equilibrio entre os lados, sem diferença significativa. Para o pico de torque na potência muscular dos atletas, nas velocidades de $180 \%$ e $240 \%$, houve diferença significativa para os extensor direito e esquerdo e no flexor direito, comparando-se bilateralmente. Portanto, os praticantes de artes marciais avaliados apresentam-se dentro do padrão, de torque e potência muscular, descrito na literatura, quando realizados os testes, mantendo, assim, o padrão extensor mais desenvolvido que o flexor, evitando, dessa forma, lesões músculo-esqueléticas.

Palavras-chave: equilíbrio muscular; taekwondo; kick boxing; potência muscular.

ABSTRACT: The martial arts Taekwondo and Kick Boxing use the kick during competition. The knee joint provides mobility and stability but is constantly moving and under stress. Biomechanical analysis in martial arts seeks to understand and quantify the mechanical overload imposed on the locomotive device, using isokinetic evaluation. This study consists of the biomechanical analysis of the knee extensor and flexor with the isokinetic dynamometer. The relationship of agonist/antagonist knee muscles in torque production was verified at the speed of $60 \% \mathrm{~s}$ and compared to the peak torque in the athletes' muscle power at the speeds of $180 \% \mathrm{~s}$ and $240 \% \mathrm{~s}$. In the agonist and antagonistic knee muscles, at the speed of $60 \%$, the average was $51.02 \%$ for the left knee and $53.43 \%$ for the right knee; however, the difference in balance between the sides was not significant. For the peak torque of the athletes' muscle power at speeds of $180 \% \mathrm{~s}$ and $240 \%$, a significant difference was found for right and left extensor and left flexor, compared bilaterally. The evaluated martial arts athletes were within standard torque and power described in literature when they did the test maintaining the more developed extensor, preventing musculoskeletal injuries.

Keywords: muscle balance; taekwondo; kick boxing; power muscle.

\footnotetext{
${ }^{1}$ Doutoranda em Engenharia Biomédica - Universidade do Vale do Paraíba - Univap. E-mails: susanetkd@hotmail.com; fegonzaganapoleone@yahoo.com.br.

${ }^{2}$ Mestre em Engenharia Biomédica - Univap. E-mail: lelinda@yahoo.com.br.

${ }^{3}$ Doutor em Biologia - Universidade Estadual do Norte Fluminense Darcy Ribeiro e Professor/Pesquisador da Univap. E-mail: nsoares@univap.br.

${ }^{4} \mathrm{PhD}$. in Computer Science - UCLA e Professor da Univap. E-mail: alderico@univap.br.

${ }^{5}$ Doutor em Ciências - UNIFESP-EPM e Professor/Pesquisador da Univap. E-mail: ralo@univap.br.
} 


\section{INTRODUÇÃO}

O estudo biomecânico dos músculos extensores e flexores do joelho, por meio da avaliação isocinética, pelo aparelho dinamômetro consiste em avaliar a quantidade de força externa e, consequentemente, interna, que estão atuando sobre um corpo, sendo de fundamental importância para a determinação do sentido do movimento (FONSECA et al., 2003).

Desta forma, a avaliação isocinética tem sido utilizada nas últimas três décadas como método para se determinar o padrão funcional da força e da relação agonista/antagonista para identificar 0 equilíbrio muscular (TERRARI; GREVE; AMATUZI, 2001).

De especial interesse, para este estudo, as modalidades Taekwondo (TKD) e Kick Boxing (KB) são artes marciais com pouca atenção para 0 desempenho muscular dos extensores e flexores do joelho, sendo os músculos mais recrutados para praticante dessas artes marciais.

O TKD, que é, oficialmente, um esporte Olímpico desde 2000, nos Jogos de Sydney, é traduzido como "caminho dos pés e da mão através da mente" sua origem é Coreana (MOHSEN et al., 2006). Durante as competições de TKD, predominam os chutes rápidos e de alta amplitude em região de tronco e cabeça do adversário, exigindo, assim, uma intensa utilização dos músculos extensores e flexores do joelho (MOHSEN et al., 2006; BEIS et al., 2001). Por outro lado, - KB, que tem como significado "chutar boxeando", é de origem americana, permite um contato total, entre os adversários, durante a competição. Vem sendo considerado um esporte de alta popularidade em todo o mundo
(GARTLAND; MALIK; LOVELL, 2001). Durante a prática de $\mathrm{KB}$, os atletas executam e sofrem diversos golpes, tais como socos, cotoveladas, chutes e joelhadas (ZORELLO, 2008).

Entretanto, até o presente momento, foi encontrado, na literatura, pouca informação com o uso da dinamometria isocinética, para a avaliação muscular de praticantes de artes marciais.

Sendo assim, a proposta deste estudo constitui-se em analisar atletas de TKD e $\mathrm{KB}$, comparando variáveis isocinéticas do joelho, tais como potência muscular, e a relação de equilíbrio agonista/antagonista em praticantes de artes marciais.

\section{MATERIAL E MÉTODO}

O presente estudo teve aprovação do Comitê de Ética do Instituto de Pesquisa \& Desenvolvimento da Univap, pelo protocolo nํ H150/CEP/2008 UNIVAP. Os testes foram realizados no laboratório de Biodinâmica da Universidade do Vale do Paraíba.

Neste estudo, foram avaliados 10 atletas do sexo masculino, com idade média de $17.2 \pm 3$ anos, altura $1.77 \pm 10 \mathrm{~cm}$, peso $67 \pm 10 \mathrm{~kg}$ e $21.7 \pm 3 \mathrm{IMC}$, e a média do tempo de treinamento variando de $5 \pm 4$ anos, sendo avaliado, bilateralmente, o membro inferior. Esses indivíduos não possuiam qualquer lesão músculo-esquelética e estavam aptos a realizar os testes propostos.

Sendo o critério de inclusão praticar artes marciais de duas a três vezes por semana e treinar para competição, e tendo como exclusão treinar apenas por lazer, e ter algum tipo de lesão muscular nos últimos seis meses. 
Cada atleta apresentou um termo de consentimento, livre e esclarecido, sendo que os menores de idade foram autorizados pelos pais. Realizou-se uma anamnese, contendo os dados pessoais e clínicos relativos à situação de cada atleta.

\subsection{Instrumento}

O dinamômetro isocinético computadorizado da Biodex foi utilizado para realizar o movimento de extensão e flexão do joelho, com as mesmas velocidades para todos os atletas, mensurando-se, assim, o pico de torque dos atletas.

Trata-se de um equipamento do modelo Biodex Multi-joint System 3, da BIODEX MEDICAL SYSTEMS INC.

\subsection{Posicionamento}

Antes de iniciarem os testes, os atletas realizarão um aquecimento de 10 minutos, em uma bicicleta ergométrica, com carga moderada.

Os atletas foram posicionados na cadeira do dinamômetro, cujo encosto foi fixado em $85^{\circ}$. O eixo do dinamômetro foi alinhado ao eixo de rotação do joelho, no epicôndilo lateral do fêmur. Os voluntários foram estabilizados, na cadeira, com cintos presos à pelve, ao tronco e à coxa a ser avaliada.

A correção da gravidade, no dinamômetro, foi realizada de acordo com as especificações do manual do equipamento (Biodex Medical System 3, Manual Applications/Operations). Para essa correção, o membro avaliado foi posicionado em extensão, local de maior atuação da gravidade, e o software do equipamento realizou o cálculo do valor desconsiderado, durante o teste.

\subsection{Procedimento no dinamômetro isoci- nético}

Os testes foram realizados, no modo isocinético concêntrico/concêntrico de flexão/extensão do joelho bilateralmente, com 5 repetições contínuas, recíprocas verdadeiras para flexão/extensão do joelho, nas diferentes velocidades angulares, sendo $60^{\circ}, 180^{\circ}$ e $240^{\circ}$, com intervalo de $180 \mathrm{~s}$, entre cada velocidade analisada. Os voluntários foram instruídos a fazer o máximo de força com velocidade, durante o teste, sendo estimulado tanto visualmente, por intermédio da tela do computador, quanto verbalmente.

\subsection{Análise dos dados}

Os dados obtidos, nas coletas com o dinamômetro isocinético Biodex Medical Systems 3, foram transportados para planilhas do Microsoft Excel, e, em seguida, foram selecionados os picos de torque flexor e extensor de cada repetição, e considerado apenas o maior, dentre os cinco valores, para cada atleta, em cada velocidade analisada, e para ambos os lados.

Realizou-se o cálculo da relação agonista/antagonista, sendo este a razão entre pico de torque extensor, dividido pelo flexor e multiplicado por cem, para a velocidade de $60 \%$ s. Com esse cálculo, obteve-se o equíbrio muscular. E, para analisar a potência muscular, foram utilizadas as velocidades $180 \%$ e $240 \%$, selecionando-se os maiores picos obtidos pela planilha do excel (TERRARI; GREVE; AMATUZI, 2001). 
Foram calculadas as médias e desvios padrões, entre as atletas, e esses resultados foram expressos na forma de tabelas e gráficos.

Para a análise estatística dos dados, utilizou-se o teste t-Student, pareado no programa Bio Estat 4.0, para comparação das diferentes velocidades de flexão e extensão do joelho, sendo o valor significativo $p \leq 0.05$.

\section{RESULTADOS}

Os resultados podem ser visualizados nas Tabelas 1 e 2 e na Fig. 1.

\section{Tabela 1 - Média e Desvio Padrão do equilíbrio muscular dos atletas}

\begin{tabular}{ccc}
\hline & \multicolumn{2}{c}{ EQUILÍBRIO MUSCULAR } \\
& Esquerdo & Direito \\
\hline Média & $51.02 \%$ & $53.43 \%$ \\
Desvio Padrão & 5.94 & 5.24 \\
\hline
\end{tabular}

Tabela 2 - Pico de torque dos músculos extensores e flexores $\left(180^{\circ}, 240^{\circ} \mathrm{Nm}\right)$

\begin{tabular}{ccccccccc}
\hline & \multicolumn{2}{c}{ Extensor $\left(\mathbf{1 8 0}^{\circ}\right)$} & \multicolumn{2}{c}{ Flexor $\left(\mathbf{1 8 0}^{\circ}\right)$} & \multicolumn{2}{c}{ Extensor $\left(\mathbf{2 4 0}^{\circ}\right)$} & \multicolumn{2}{c}{ Flexor $\left(\mathbf{2 4 0}^{\circ}\right)$} \\
& Esq & Dir & Esq & Dir & Esq & Dir & Esq & Dir \\
\hline MÉDIA & 125.6 & 139.95 & 91.76 & 87.83 & 112.75 & 122.71 & 84.75 & 100.75 \\
DESVIO PADRÃO & 28.18 & 29.69 & 16.26 & 19.29 & 21.87 & 28.07 & 19.64 & 17.52 \\
\hline
\end{tabular}

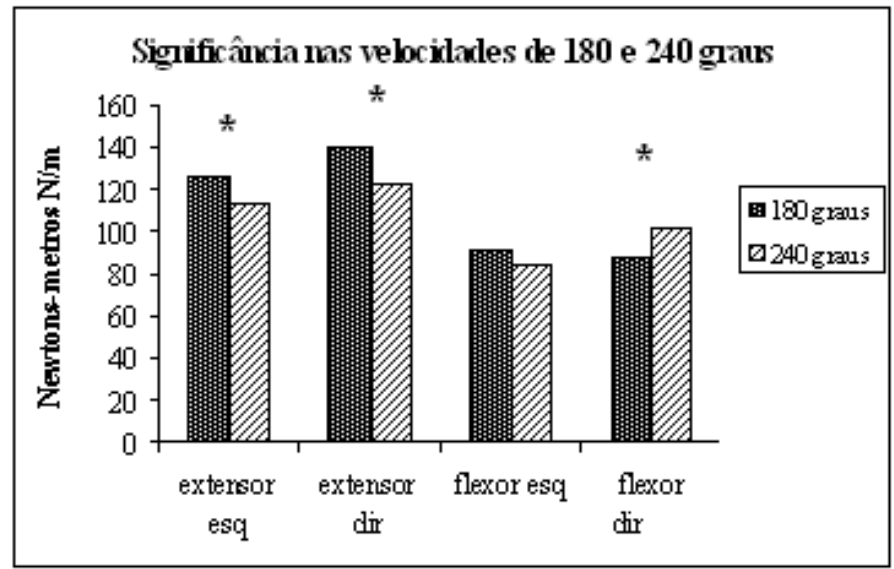

Fig. 1 - Comparação dos valores de significância, para as velocidades de 180 e 240 graus, mostrando que houve diferença significativa para os extensor direito e esquerdo e no flexor direito. 


\section{DISCUSSÃO}

Pesquisas envolvendo estudos desportivos analisando o equilíbrio muscular agonista/antagonista e pico de torque, ao longo dos anos, têm sido de grande interesse devido a preocupação em analisar dados que apresentem o equilíbrio muscular na articulação do joelho, bem como a relação do desequilíbrio muscular que ocasionam as lesões. Porém, são escassos os estudos publicados que forneçam dados relativos às consequências da prática de artes marciais. E, como não existe nenhum protocolo padronizado para avaliar a força dos atletas, torna-se difícil comparar os resultados com os diversos estudos.

O conceito de equilíbrio funcional musculotendíneo reflete um parâmetro importante na adequada realização da prática de esportes. $\mathrm{O}$ dinamômetro isocinético quantifica precisamente o pico de torque, como também oferece dados de comparação do lado dominante e não dominante, do membro contra-lateral e homolateral de grupos musculares (ANDRADE; FLEURY, 2003). Segundo alguns autores, uma diferença de até 10\% entre membros inferiores pode ser considerada normal, ao se considerar a dominância normal.

A verificação do desequilíbrio agonista/antagonista é calculada por um dado percentual, que é obtido por meio da divisão do torque máximo do flexor pelo extensor, e multiplicado por $100 \%$.

Vários estudos quantificaram o torque extensor e flexor do joelho em diferentes populações (SCHENEIDER; RODRIGUES; MEYER, 2002; FONSECA et al., 2003; UGRINOWITSCH et al., 2000). Os principais resultados indicaram que o torque extensor predominou sobre o torque flexor, obtendo razão agonista/antagonista de cerca de $50 \%$ (CESARELLI; BIFULCO; BRACALE, 1999).

Os valores resultantes deste estudo, referentes à relação da musculatura agonista/antagonista do joelho, na velocidade de $60 \%$ s, mostrou-se um pouco abaixo dos valores mínimos descritos na literatura como padrão de normalidade. O estudo de Machado et al. (2009) analisou variáveis isocinéticas por meio do dinamômetro de atletas de TKD e KB, sendo constatado um equilíbrio muscular na velocidade angular de $60 \%$, porém sugeriuse uma incidência à lesão muscular alta, devido a exigência muscular durante os treinamentos $e$ as competições, sendo necessário para 0 praticante de artes marciais, um aumento da força muscular para o grupo flexores de joelho.

No estudo de Carvalho e Cabri (2007), com 245 futebolistas, na velocidade de $60 \%$, os resultados sugerem que todos os sujeitos da amostra apresentaram equilíbrio funcional em nível da articulação do joelho, tendo $50-60 \%$.

O conjunto músculo-articular, muito solicitado nas modalidades, deve ser cuidadosamente avaliado devido à frequência de lesões ocasionadas por desequilíbrio muscular. Os músculos estabilizadores do joelho devem ter resistência equilibrada entre a esquerda e a direita, e relação entre agonista/antagonista, resistência de todo o membro (músculos proximais e distais) e relação variável entre o estímulo e a resposta (velocidade de contrair e relaxar). O desequilíbrio entre a força dos músculos ísquio-tibiais e do quadríceps sugere não ultrapassar o potencial de força do quadríceps mais do que $10 \%$, em relação aos músculos ísquiostibiais, para proteger das lesões (PAIXÃO; 
AKUTSU; PINTO, 2004).

O descuido no treinamento de força da musculatura ísquio-tibial pode reduzir a coativação dos agonistas e antagonistas como resposta à superioridade de força do quadríceps. Tais atividades reduzidas do antagonista diminuem a força de estabilização total disponível da articulação durante a carga de esforço a maior risco de lesões (MOHSEN; PIETER, 2004).

O presente estudo também analisou 0 pico de torque na potência muscular dos praticantes, nas velocidades de $180 \% \mathrm{~s}$ e $240 \%$, que são consideradas velocidades rápidas, sendo de acordo com o estudo de Mohsen et al. (2004) que confirmam que o desequilibrio dessas musculaturas aumenta a probabilidade de lesões musculares.

A potência muscular é altamente dependente da força, sendo caracterizada como a integração entre força e velocidade. $O$ pico de torque representa o ponto de maior torque na amplitude de movimento, o torque e a velocidade angular de movimento são grandezas inversamente proporcionais, ou seja, quanto menor a velocidade angular realizada, maior será o torque; quanto maior a velocidade, menor o torque (TERRERI et al., 1999; MOHAMED; PERRY; HISLOP, 2002).

Machado et al. (2010) analisaram as modalidade de TKD e KB, por meio da eletromiográfia, constatando que os atletas de KB tem um maior recrutamento de fibras musculares, compados aos atletas de TKD, sendo o KB uma modalidade que exige força muscular e o TKD velocidade à execução dos golpes.

Sabe-se que, nos dias de hoje, o interesse em se prevenir lesões, por meio da identificação precoce da insuficiência do grupo muscular, ou de desequilíbrio entre músculos antagonistas de uma articulação, levando em consideração a articulação do joelho, seriam os flexores, e, para que isso seja possível, torna-se imprescindível o uso de aparelhos, como o dinamômetro, que avalia força muscular que é de grande interesse clínico para 0 desempenho muscular (MACHADO et al., 2009; ROSENE; FOGARTY; MAHAFFEY, 2005; PIETER, 2005; PIETER; LUIGI; BERCADES; KIM, 2006).

Por isso, acredita-se que treinos, com cargas e velocidades, é uma forma de estimulação para adaptações do organismo diante às exigências dinâmicas, permitindo a reestruturação morfológica que possibilita melhoras funcionais aos atletas.

\section{CONCLUSÃO}

A utilização da avaliação isocinética, em atletas de arte marciais, para analisar a função muscular, é pouco estudada, com um pequeno número de publicações disponíveis na literatura científica. Portanto, conclui-se que, neste estudo, os atletas de artes marciais avaliados apresentam-se dentro do padrão de torque e potência descritos na literatura, quando analisados, com teste na velocidade mais alta $(180 \% \mathrm{~s}$ e $240 \% \mathrm{~s})$, mantendo 0 padrão extensor mais desenvolvido. O mesmo não foi observado na velocidade de $60 \%$, visto que a relação entre a musculatura agonista e antagonista encontrou-se, com média inferior ao valor mínimo descrito; no entanto, há um equilíbrio entre os lados, sem diferença significativa, sugerindo que haja um treinamento de compensação, trabalhando os dois membros, para que não haja, assim, desequilíbrios musculares entre eles. 
Ressalta-se, também, a importância da conscientização profissional e do atleta, que o trabalho de condicionamento físico, bem como a complementação com exercícios de reforço muscular e alongamentos, são fundamentais à prevenção de lesões.

\section{REFERÊNCIAS}

ANDRADE, M. S.; FLEURY, A. M. Risco de lesão na articulação do joelho no ciclismo Indoor. São Paulo: USP; Centro de Estudos de Fisiologia do Exercício, 2003.

BEIS, K. et al. Taekwondo competition injuries in Greek young and adult athletes. Eur. j. sports traumol. rel. res., v. 23, p. 130136, 2001.

CARVALHO, P.; CABRI, J. Avaliação Isocinética da força dos músculos da coxa em futebolistas. Rev. Port. fisioter. desp., v.1, p.4-12, 2007.

CESARELLI, M.; BIFULCO, P.; BRACALE, M.: Quadriceps muscle activation in anterior knee pain during in isokinetic exercise. Med. eng. phys., v. 21, p. 469-478. 1999.

FONSECA, S. T. et al. Perfil de dinamometria isocinética da articulação do joelho de jogadores da seleção brasileira infanto-juvenil de voleibol masculino. In: CONGRESSO BRASILEIRO DE BIOMECÂNICA, 10. Ouro Preto, 2003; Anais.... p. 339-342. 2003.

GARTLAND, S.; MALIK, M.; LOVELL, M. Injury and injury rates in Muay Thai kick boxing. Br. j. sports med., v. 35, p. 308-313, 2001. doi: 10.1136/bjsm.35.5.308

MACHADO, S. M. et al. Estudo comparativo de variáveis isocinéticas do joelho em atletas de taekwondo e kickboxing. Fit. perf. j., v. 8, p. 407-411, 2009.
MACHADO, S. M. et al. Biomechanical analysis of the muscular power of martial arts atheletes. Med. biol. eng. comput., v. 48, p. 573-577, 2010.

MOHAMED, O., PERRY, J., HISLOP, $\mathrm{H}$. Relationship between wire EMG activity, muscle length, and torque of the hamstrings. Clin. biomech., v. 17, n. 8, p. 569-579, 2002.

MOHSEN, K. et al. A profile of olympic taekwondo competitors. J. sports sci. med., v. 5, p. 114-112, 2006.

MOHSEN, K, PIETER, W. Injuries at a Canadian National Taekwondo Championships: a prospective study. BMC musculoskelet. disord., v. 5, p. 22, 2004.

PAIXÃO, D. O.; AKUTSU, M. L. S.; PINTO, S. S. Avaliação Isocinética da média de torque e potência em flexores e extensores de joelhos relacionando o posicionamento em campo, idade e membro dominante em atletas de futebol profissional. Reabilitar, v. 6, n. 24, p. 10-20, 2004.

PIETER, W. Martial Arts Injuries. Med Sport Sci., v. 48, p. 59-73, 2005.

PIETER, W.; BERCADES, L. T.; KIM, G. Relative Total Body Fat And Skinfold Patterning In Filipino National Combat Sport Athletes. J. sports sci. med., n. CSSI, p.3541, 2006.

ROSENE, J.; FOGARTY, T. D.; MAHAFFEY, B. L. Isokinetic hamstrings: Quadriceps ratios in intercollegiate athletes. J. athl. train., v. 36, p. 378-383, 2001.

SCHENEIDER, P.; RODRIGUES, L.; MEYER, F. Dinamometria computadorizada com metodologia de avaliação de força muscular de meninos e meninas em diferentes estágios de maturidade. Rev. paul. educ. fís., v. 16, p. 35-42, 2002. 
TERRERI, A. S. et al. Isokinetic assessment of the flexor-extensor balance in athletes with total rupture of the anterior cruciate ligament. Rev. Hosp. Clin., São Paulo, v. 54, p. 53-60, 1999.

TERRERI, A. S. A. P.; GREVE, J. M. D.; AMATUZZI, M. M. Avaliação isocinética no joelho do atleta. Rev. Bras. med. esporte, v. 7, n.5, p. 170-174, 2001.
UGRINOWITSCH, C. et al. Capacidade dos testes isocinéticos em predizer a "performance" no salto vertical em jogadores de voleibol. Rev. paul. educ. fís., v. 14, n. 2, p. 172-183, 2000.

ZORELLO, P. História do Kick Boxing. Rio de Janeiro: APKB, 1992. Disponível em: $<$ http://www.apkb.com.br/arquivos/apostilaapkb.pdf>. Acesso em: 10 mar. 2008. 